\title{
Racial and Socioeconomic Differences in Social Work and Department of Child Services Reporting Patterns for THC Positive Pregnant Women
}

\author{
Sydney DiGregory ${ }^{1}$, Anneli Cochrane ${ }^{1,2}$, Brownsyne Tucker Edmonds, ${ }^{2}$ \\ ${ }^{1}$ Indiana University School of Medicine; ${ }^{2}$ Department of Obstetrics and Gynecology
}

Background and Hypothesis: Tetrahydrocannabinol (THC) is the most commonly used illicit substance during pregnancy. Little research, most with conflicting data, exists on its impact on the developing fetus or birth outcomes. Reporting THC positive urine drug screen (UDS) to social worker (SW) has been left to provider discretion. Similarly, SW has discretion in reporting to Department of Child Services (DCS) if the newborn tests negative for drugs at birth. We hypothesize that there are racial and socioeconomic differences in reporting patterns that result in non-white and publicly insured patients being reported to SW or DCS at higher rates than white or privately insured patients.

Methods: Data were collected via Cerner and CareWeb chart abstraction of patients who delivered at IU Health hospitals between 1/1/16 to 12/31/17 and tested positive for THC, but no other drugs, during any stage of pregnancy. Data was entered into the secure and encrypted Redcap database. Demographic variables included mother's age, race, ethnicity, insurance type, and number of previous pregnancies. Descriptive statistics were calculated for demographics by overall, SW, and DCS referral. p-values were calculated using Fisher's Exact Test for small sample sizes and considered significant at the 0.05 level. The effects of age and number of previous pregnancies were analyzed using two-sample t-test for equal variances.

Results: 152 patients met inclusion criteria for the analysis. The study population was mostly white (68.4\%) and black (30.3\%). Their insurance type was $74.3 \%$ public, $17.8 \%$ private, and $7.9 \%$ uninsured. The vast majority of cases were referred to SW (90.8\%) and DCS (87.7\%). Neither patient race nor insurance were associated with referral to SW $(p=.80, p=.79)$ or DCS $(p=.30, p=.25)$.

Conclusion: There was no statistically significant association between demographic characteristics and referral patterns, though the study may be underpowered due to low nonreferral rate. Addition of 2018-2019 data are planned. 